\title{
Analytics-driven health care growing in Ontario
}

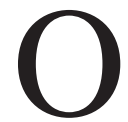

ntario data specialists are dusting off reams of disparate and disconnected health data, integrating it with new sources of "big data" from genomics, and harnessing it all to help shape reform in clinical care, public health strategy and healthsystem financing.

"The link between analytics and policy has never been stronger," says Alison Blair, interim director of the Health Analytics Branch at the Ontario Ministry of Health and Long-Term Care.

For evidence, Blair says the health ministry's two biggest reform initiatives are founded upon information integrated from a broad array of formerly isolated databases. One initiative is to integrate family care providers, specialists, hospitals, long-term care, home care and other community supports. The other is exploring how to replace unconditional funding grants to hospitals with a system where pay is based on the quality and quantity of patient care the hospitals actually deliver.

Most health data systems were built for managing acute care, but now health providers want a more complete picture of "patients' end-to-end journeys," says Jeanette Munshaw, director of the ministry's Health Data Branch. "To do that, we need to integrate myriad sources of data," she adds.

Munshaw's team is currently drawing upon records from 48 hospitals and 15 provincial health databases to identify the price of treating high-cost patients, which will allow the ministry to refine payments.

Soon, there will also be huge volumes of additional health data flowing from the 10000 electronic medical record (EMR) systems used by Ontario physicians, notes Dr. Karen Tu, who leads the development of the Electronic Medical Record Administrative Data Linked Database (EMRALD) at the Institute for Clinical and Evaluative Sciences in Toronto, Ontario.

Health information, Tu believes, is poised for "a great leap forward" as EMR data come online. By linking EMRs with databases from other sources - the Canadian Institute for

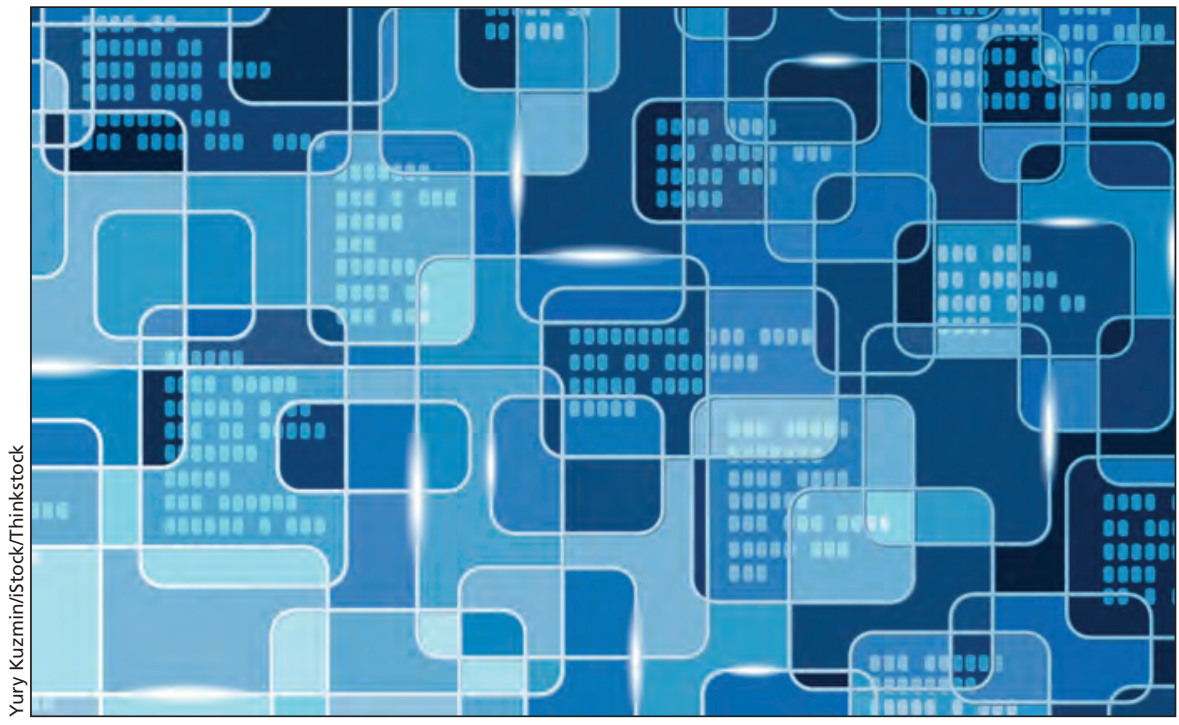

Health data specialists are integrating data from various sources to guide health care decisions.

Health Information, the Ontario Drug Benefit Program, the Ontario Health Insurance Plan and others - the EMRALD database can compile patient health histories that are more comprehensive than ever seen before, says Tu.

Studies published using EMRALD data have focused on physicians' performance on quality indicators for diabetes and ischemic heart disease care, wait time for appointments with specialists following physician referrals, the prevalence of obesity in children and the impact of adopting an EMR on physician billings.

The health system is also moving toward linking to data on people's social circumstances, housing, and food and income security, says Walter Wodchis, leader of the Health System Performance Research Network. Meanwhile, as part of a study of 225000 people, the province is developing databanks of biological specimens from individuals that can be used to track long-term health outcomes by linking genetic and blood profiles with health care events.

"This allows for very deep data," says Wodchis. Using big data more to segment and group people with similar and distinct needs, he adds, will expedite more effective health reforms.

Health care delivery is also being refashioned by data-integration innova- tions, notes Wendy Gerrie, director of Integrated Decision Support Services at Hamilton Health Sciences in Ontario. As she enthused at a recent data analytics conference in Toronto "total patient data capture is emerging."

Gerrie developed a system that links patient information - demographics, clinical characterizations, emergency visits, hospital admissions and discharges - from a plethora of formerly disconnected databases. It is capable of reaching beyond health care into data from social service agencies. "The data we're integrating is much more than just different types of hospital information," Gerrie explains. "We're bringing in housing data, for example, because we've identified that as an issue relevant to health care."

The system, operated by the Hamilton Niagara Haldimand Brant Local Health Integration Network, is being adopted by three other local hospital networks in southern Ontario. Capable of producing 250 different types of reports, the system lets users "drill down to see where the patients are coming from," says Gerrie. "We've begun to identify the high-user population. And once they are identified, a shared care plan is put in place." - Paul Christopher Webster, Toronto, Ont.

CMAJ 2014. DOI:10.1503/cmaj.109-4693 